\title{
PA-089 CHILD PROTECTION AND DEVELOPMENT: ADDRESSING THE PROBLEMS OF HIVIAIDS ORPHANS - A CASE STUDY IN BAHIR DAR TOWN, ETHIOPIA
}

Ayele Tamene. Bahir Dar University, Ethiopia

10.1136/bmjgh-2016-000260.121

Background HIV/AIDS has continued to be a world social, economic and political threat. Recent findings indicated that currently 34 million people are living with HIV/AIDS. Sub-Saharan countries are particularly vulnerable to this pandemic. Ethiopia's HIV/AIDS epidemic pattern marked regional variations across urban and rural areas. Ethiopia which is one of the largest populations in Africa has the highest number of orphans. The proportion of orphan children due to AIDS is also alarmingly increasing in this country. It increased from $26 \%$ to $43 \%$ 
in Ethiopia in 2001 to 2010. The situation of AIDS orphans have become a serious problem in Ethiopia.

Methods A qualitative method of study, particularly a phenomenological approach, was used to guide the study. Data were collected through interviews, focus group discussions and case studies. In addition, a secondary review of documents such as reports, annual and strategic plans was done.

Results The study indicated that there are variations in the number of orphans across kebeles of the town. The Shinbit kebele has the highest number of orphans, both male and female. In Bahir Dar various forms of service are rendered to orphans living with HIV/AIDS such as psycho-social support, educational and medical support, economic strengthening through guardians, home to home support, legal support, vocational and skill development training.

Conclusions In Bahir Dar, various forms of service are rendered to HIV/AIDS orphans such as psycho-social support, educational and medical support, economic strengthening through the guardians, legal support, vocational and skill development training. These methods of addressing HIV/ AIDS orphans form a fragmented, only need-based approach, and are far from a right-based approach as they lack institutional networking, sustainability and community ownership. Therefore, in order to meet one of the objectives of the Cross-Cutting Sectors Development Plan of the Growth and Transformation Plan, it is recommended to address the multifaceted problems of AIDS orphans in an integrated and sustainable way. 